Document downloaded from:

http://hdl.handle.net/10251/145866

This paper must be cited as:

Torregrosa, AJ.; Piqueras, P.; Sanchis-Pacheco, EJ.; Guilain, S.; Dubarry, M. (08-1). Assessment of acoustic reciprocity and conservativeness in exhaust aftertreatment systems. Journal of Sound and Vibration. 436:46-61. https://doi.org/10.1016/j.jsv.2018.08.032

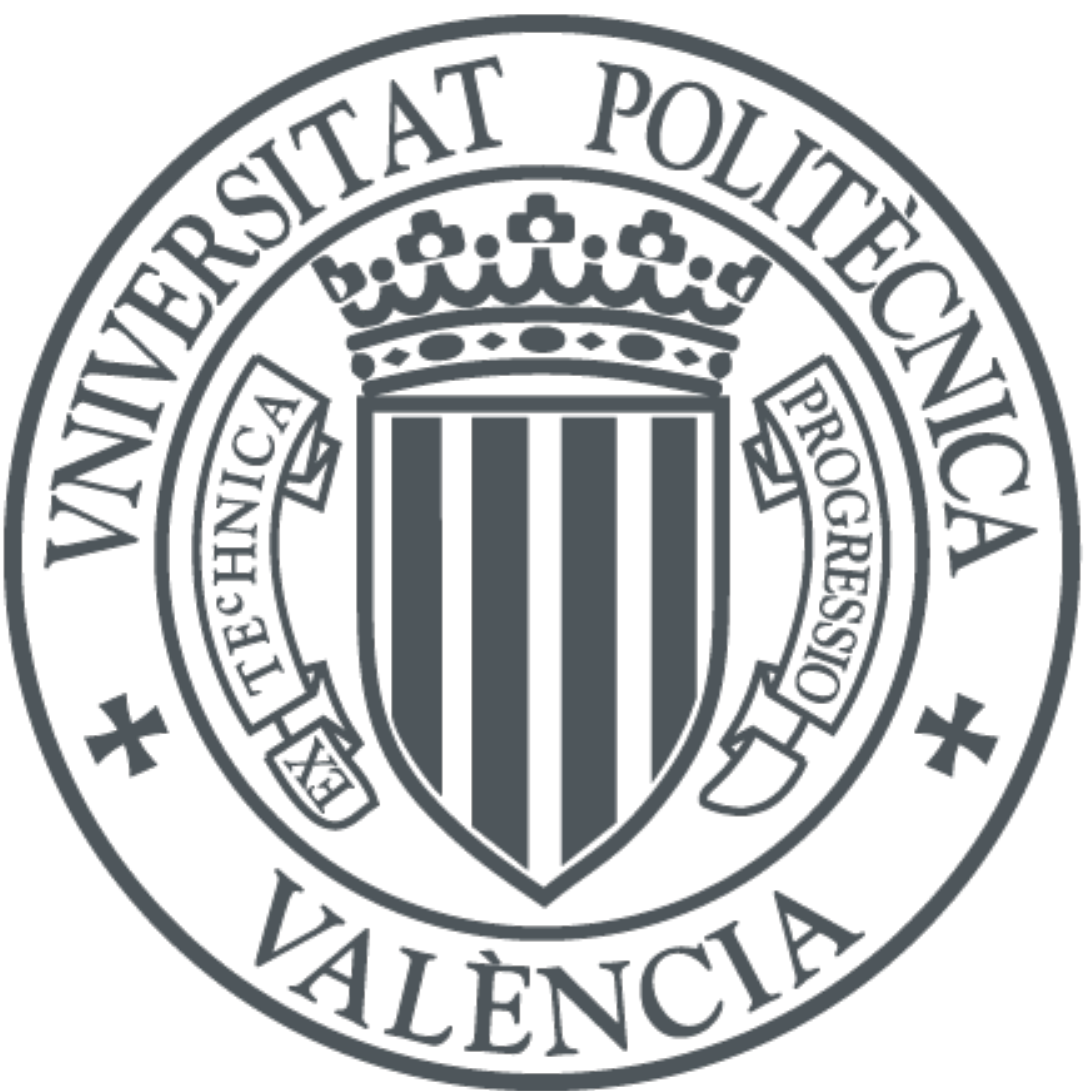

The final publication is available at

https://doi.org/10.1016/j.jsv.2018.08.032

Copyright Elsevier

Additional Information 


\title{
Assessment of acoustic reciprocity and conservativeness in exhaust aftertreatment systems
}

\author{
A. J. Torregrosa ${ }^{\mathrm{a}}$, P. Piqueras ${ }^{\mathrm{a}, *}$, E. J. Sanchis ${ }^{\mathrm{a}}$, S. Guilain ${ }^{\mathrm{b}}$, M. Dubarry $^{\mathrm{b}}$ \\ ${ }^{a}$ CMT-Motores Térmicos, Universitat Politècnica de València, Camino de Vera s/n, 46022 Valencia, Spain. \\ ${ }^{b}$ Renault SA, 1 alle Cornuel, 91510 Lardy, France.
}

\begin{abstract}
Tightening emission standards limiting gas and aerosol emissions from internal combustion engines have led to the extensive use of exhaust aftertreatment systems (EATS) with different chemical functions as a solution to meet standards requirements. Incidentally, the placement of aftertreatment monolithic devices into the exhaust line also plays a key role on the exhaust noise emission. Their presence disturbs the pattern of the pressure waves and sets the boundary conditions for the silencer design. The impact of the EATS on wave transmission can be analyzed by means of the transmission or scattering matrix. The present work discusses the implications of acoustic reciprocity and conservativeness on the definition of the scattering matrix elements. The fulfillment of these properties in real operating conditions was evaluated against a set of experimental data obtained for several exhaust aftertreatment monolithic bricks in an impulse test rig. The influence of different excitation amplitudes and superimposed mean flows was also considered. Once it was shown that the devices are reciprocal, the need to account for dissipation phenomena was evidenced. Finally, the application of reciprocity and conservativeness together with dissipation provided simple expressions allowing to predict the response of the EATS in the inverse direction, i.e. from outlet to inlet, from the transmission and reflection properties obtained in the direct direction. Thus, the proposed procedure becomes useful to reduce both the required number of tests and the gas dynamics modelling work in methodologies driven to assess the acoustic response of EATS based on the use of experimental and computational tools.

Keywords: Internal combustion engine, exhaust aftertreatment system, acoustics, scattering matrix, reciprocity, dissipation
\end{abstract}

${ }^{*}$ Corresponding author. Tel.: +34 963877650 , fax: +34963877659 .

Email address: pedpicab@mot.upv.es (P. Piqueras) 


\section{Nomenclature}

\begin{tabular}{|c|c|c|c|}
\hline$a$ & speed of sound & $Y$ & impedance coefficient \\
\hline$a_{k}$ & main Fourier series constant & $\mathbf{Y}$ & impedance matrix \\
\hline$a_{k}^{\prime}$ & deviation Fourier series constant & Greek letters & \\
\hline$A$ & cross-section area & $\alpha$ & honeycomb cell size \\
\hline$b_{k}$ & main Fourier series constant & $\delta$ & dissipation term \\
\hline$b_{k}^{\prime}$ & deviation Fourier series constant & $\Delta$ & transfer matrix determinant \\
\hline$D$ & monolith diameter & $\gamma$ & specific heat ratio \\
\hline$f$ & frequency & $\pi$ & characteristic periods ratio \\
\hline \multirow[t]{2}{*}{$F$} & parameterized real or imaginary part & $\sigma$ & cell density \\
\hline & of the scattering matrix coefficients & $\tau$ & characteristic period of main Fourier series \\
\hline$H_{i j}$ & transmission matrix element & $\tau^{\prime}$ & characteristic period of deviation Fourier series \\
\hline $\mathbf{H}$ & transmission matrix & $\phi$ & argument of scattering matrix element \\
\hline $\operatorname{Im}$ & imaginary part & Acronyms & \\
\hline$L$ & monolith length & $1 \mathrm{D}$ & one-dimensional \\
\hline$n$ & Fourier series order & $2 \mathrm{D}$ & bi-dimensional \\
\hline$p_{0}$ & unperturbed medium pressure & $3 \mathrm{D}$ & three-dimensional \\
\hline$p_{\text {comp }}$ & composed pressure & DOC & diesel oxidation catalyst \\
\hline$p_{\text {inc }}$ & incident pressure & DPF & diesel particulate filter \\
\hline$p_{\text {ref }}$ & reflected pressure & EATS & exhaust aftertreatment system \\
\hline$P$ & acoustic pressure & TWC & three-way catalyst \\
\hline$P^{+}$ & forward pressure component & Subscripts & \\
\hline$P^{-}$ & backward pressure component & 1 & referred to inlet conditions \\
\hline$r_{1}$ & direct reflection coefficient & 2 & referred to outlet conditions \\
\hline$r_{2}$ & inverse reflection coefficient & $b$ & baseline operating point \\
\hline$R^{2}$ & coefficient of determination & $\operatorname{dir}$ & direct test \\
\hline $\operatorname{Re}$ & real part & $\exp$ & referred to experimental data \\
\hline$t$ & time & in & inlet \\
\hline$t_{12}$ & direct transmission coefficient & inv & inverse test \\
\hline$t_{21}$ & inverse transmission coefficient & $j$ & real or imaginary part of $t$ or $r$ coefficient \\
\hline$T_{i j}$ & transfer matrix term & $\bmod$ & fluid-dynamic model \\
\hline $\mathbf{T}$ & transfer matrix & $p$ & arbitrary operating point \\
\hline$V$ & mass velocity & $\mathrm{pr}$ & prediction \\
\hline$w_{w}$ & monolith channel wall thickness & out & outlet \\
\hline
\end{tabular}




\section{Introduction}

Emission standards applied to ground transport power plants have been progressively tightened along the last decades $[1,2]$ as a way to make internal combustion engines more respectful with environment and human health. Although the great improvements achieved in emissions reduction cannot be completely explained without mentioning advances in air management, injection and combustion processes, aiming at the reduction of engine raw emissions, the fulfillment of current and upcoming pollutant regulations demands the use of aftertreatment devices for exhaust emissions abatement [3]. Consequently, the presence of these devices involves the need to account for its influence on the noise transmitted by the exhaust line.

The reduction of tailpipe emitted noise is traditionally addressed by means of silencers [4]. The properties of these devices are well established for both reactive mufflers, whose purpose is to reflect the acoustic energy back to the source, and dissipative mufflers, in which the acoustic energy is dissipated.

It is apparent that the presence of the aftertreatment monoliths along the exhaust line complements, and therefore affects, the design of the silencer, which is subject to less demanding attenuation targets due to noise damping at the aftertreatment [5]. In this sense, the muffler design should not only adapt to the upstream boundary condition imposed by the presence of the monolith, but also take advantage for volume and pressure drop reduction [6].

Honeycomb monoliths disturb the unsteady wave dynamics in the exhaust line [7] and produce a certain attenuation on the noise emitted by the exhaust orifice [8]. The effect observed, however, differs between catalytic reactors, which are flow-through monoliths [9], and particulate filters, which are characterized by a wall-flow monolith structure [10] where the flow is forced to pass through the porous medium. The variable properties of the porous substrate play a major role in the acoustic response of the device, which depends on the porous wall permeability and on the particulate layer properties [11]. In addition, the main features of the reactive response are also dependent on the soot loading and distribution along the inlet channels [12].

The acoustic modelling of exhaust aftertreatment systems (EATS) covers a wide spectrum of models in terms of solution approach, i.e. linear [8] and non-linear methods [12], spatial resolution ranging from 3D [6] to phenomenological lumped models [13], with models of intermediate complexity based on simplified 3D-cell [14] and 1D/2D approaches [10]. Different numerical schemes have been also considered, from finite element methods [15, 16] focused on the analysis of wave propagation along the monolith channels, to boundary element methods to account for the three-dimensional effects in the inlet and outlet canning volumes [17]. While all these theoretical tools are fundamental for the analysis and optimization of the acoustic response of EATS, their validity strongly depends on the availability of reliable experimental data. The variety of techniques proposed to obtain forward and backward pressure perturbations at the inlet and outlet of any device $[8,18]$ demand criteria to complement the error analysis as a way to verify the measurement consistency. In addition, procedures able to reduce the required number of tests, 
both experimental or computational, are also of great interest. The analysis of acoustic properties, such as reciprocity or conservativeness of a system, contributes to this purpose. Reciprocity $[19,20]$ involves that the response at a given location to a disturbance applied at another point is identical to the response that would be obtained if the positions were exchanged [21]. Conservativeness means that the energy of the waves transmitted and reflected by a system is the same as the energy of the waves incident to the system. Likewise, describing the acoustic behaviour of the devices with their characteristic matrices enables the mathematical definition of reciprocity and conservativeness [22]. Besides verification, these properties have also found important practical applications in complex systems, as in the case presented by Easwaran et al. [23] for multipole systems.

The present paper assesses the applicability of reciprocity and conservativeness to different honeycomb monolithic aftertreatment systems and their potential use in real-life cases. The formulation of the reciprocity and conservation properties is firstly presented in terms of the scattering matrix elements. Then, these properties are applied to the prediction of the acoustic response of a set of EATS devices. The theoretical results are analyzed by comparison against experimental data obtained in an impulse test rig where the aftertreatment systems were subject to a variety of excitations of different amplitude and also considering the impact of a superimposed mean flow. Additionally to the tests of isolated monoliths, tests were performed on systems including both a diesel oxidation catalyst (DOC) and a wall-flow particulate filter (DPF). Thus, it was possible to assess the validity of the discussed acoustic properties taking into account the combined effect of flow-through and wall-flow monoliths. The fulfillment of the reciprocity property was studied by means of the prediction of the inverse transmission coefficient from the direct transmission one. In addition, the dissipative effects of the system were discussed by analyzing the relation between the modules of the direct and inverse reflection coefficients and the corresponding dissipation terms. In this way, the expressions obtained for the prediction of inverse transmission matrix elements become a useful tool to complement methodologies for the characterization of EATS traditionally based on the application of conventional experimental and computational tools.

\section{Experimental methodology}

The discussion on the fulfillment of the reciprocity and conservativeness properties is supported by the experimental characterization of the acoustic response of different aftertreatment systems. Tests were conducted in a gas stand whose design is based on the impulse method [18]. The tested device is subject to an isolated pressure pulse of controlled amplitude and duration. The reflected and transmitted pressure perturbations are registered by piezoelectric pressure sensors. Figure 1 shows the scheme of the experimental set-up and its operating principle.

The impulse method is based on the generation of a pressure pulse at room temperature by means of the aperture of an high-speed electrovalve controlling the discharge from a pressurized tank. The pressure in the tank and the duration of the electrovalve opening period set the amplitude and duration of the pulse, respectively. The generated pressure pulse, which is measured by pressure transducer 1, propagates towards the device along the inlet duct, whose 
length is properly selected to ensure that the measurement of pressure transducer 1 is not affected by the reflection travelling back from the tested device towards the electrovalve, as sketched in Figure 1.

The superposition of the incident and reflected pulses $\left(p_{\text {comp }}\right)$ is registered by pressure transducer 2 , which is placed close to the EATS. Finally, the pressure pulse transmitted downstream of the EATS is registered by pressure transducer 3. This measurement is not affected by secondary reflections from the end of the outlet duct because again the length of this duct is selected to avoid the superposition of pressure waves within the measurement window.

Such superposition of the incident and reflected pulses at the device inlet requires the determination of the reflected wave in order to characterize the acoustic response of the tested device. With that purpose, a straight duct portion is tested instead of the EATS so that the incident pressure at the inlet section can be identified. In summary, the characterization is performed according to the following steps [18]:

1. The EATS is tested with several incident pressure pulses of the same amplitude and duration (repeatability within a prescribed uncertainty below 5\%). These tests provide the composed pressure at the inlet section and the transmitted pressure at the outlet section.

2. A straight duct is tested with incident pressure pulses similar to those exciting the EATS in step 1. These tests provide the incident pressure.

3. The most coincident pair of EATS and straight duct tests are identified based on the comparison of the generated pressure registered by transducer 1 . The statistical procedure used for the selection of these tests can be found in [13]. Once the tests are selected, the incident pressure pulse $\left(p_{\text {inc }}\right)$ at the EATS inlet is defined as that measured by pressure transducer 2 in the straight duct test. This incident pressure pulse is combined with the measurement of pressure transducer 2 in the EATS test to determine the reflected pressure pulse $\left(p_{\text {ref }}\right)$ applying Eq. (1) $[24,25]$ :

$$
\left(\frac{p_{\text {ref }}}{p_{0}}\right)^{\frac{\gamma-1}{2 \gamma}}=\left(\frac{p_{\text {comp }}}{p_{0}}\right)^{\frac{\gamma-1}{2 \gamma}}-\left(\frac{p_{\text {inc }}}{p_{0}}\right)^{\frac{\gamma-1}{2 \gamma}}+1,
$$

where $p_{0}$ is the pressure of the unperturbed medium.

This process allows obtaining the scattering matrix coefficients of the EATS in one direction of excitation, so that it is repeated twice, i.e. this is done for both directions of excitation:

- The direct test consists of the excitation of the device with an incident pressure pulse travelling from the inlet to the outlet to assess the reflection at the inlet interface and the transmission from inlet to outlet.

- The inverse test consists of the excitation of the device with an incident pressure pulse travelling from the outlet to the inlet to assess the reflection at the outlet interface and the transmission from outlet to inlet.

Since the installation is fixed, change from direct to inverse test is done by reversing the EATS in the test rig. The described procedure is completed with the optional superimposition of a mean flow from inlet to outlet of the device. 
The mass flow control is performed acting on the pressure of a tank installed at the outlet of the impulse test rig. The tank works in vacuum mode in direct tests whilst its pressure is increased in inverse tests.

Figure 2 shows an example of the data provided by the impulse test rig and the post-processing procedure. The incident, transmitted and reflected pressure pulses measured in the direct and inverse tests of the system composed by DOC+DPF-2 are shown. The main geometric parameters of these devices are detailed in Table 1, which includes the characteristics of each tested EATS. Figure 2 represents the results for the baseline operating point, which is defined by an amplitude and duration of the generated pulse of $150 \mathrm{mbar}$ and $14 \mathrm{~ms}$ respectively, without any superimposed mean mass flow. This excitation was tested in all the devices. Additional operating points were tested in order to account for the influence of the convective transport. The instantaneous convective transport was assessed by changing the amplitude and duration of the excitation, whilst mean convective transport effects were evaluated with a superimposed mean mass flow of $170 \mathrm{~kg} / \mathrm{h}$. It is interesting to note that the pulse profiles provided by the impulse test rig are representative of the pressure pulses impinging on the elements placed in the exhaust cold end in realistic conditions. This was discussed by Payri et al. [18], who evaluated the modified impulse method applied in this work. According to the basis of the impulse method, the propagation of the pressure pulse along the inlet duct is non-linear. Hence, Eq. (1) is applied. Nevertheless, the non-linear development of the pressure pulse leads to the formation of an asymptotic singularity. Once the pulse reaches the asymptotic region any further non-linear development should be scarcely noticeable, both in the time and frequency domains. This occurs somewhere upstream of transducer 2 , as shown in Figure 2, and makes it possible to consider a linear method to analyze the acoustic response of the tested device.

\section{Assessment of reciprocity}

Eq. (2), which defines the reciprocity property in terms of direct and inverse transmission coefficients as presented in Appendix B, was applied to evaluate the fulfillment of reciprocity in the tested aftertreatment devices.

$$
t_{21}=\frac{Y_{1}}{Y_{2}} t_{12}
$$

Figure 3 presents the comparison between the experimental and the theoretical (i.e. computed according to Eq. (2) inverse transmission coefficient $\left(t_{21}\right)$. The modulus and the argument obtained in the direct and inverse tests are shown for the three tested devices, which include systems consisting of both flow-through and wall-flow monoliths. In all cases, the excitation is the baseline one, i.e. a pressure pulse of 150 mbar in amplitude and 14 ms in duration without superimposed mass flow. The experimental coefficients, which are represented by the black lines, were parameterized by the two-step Fourier series procedure described in [13].

Good agreement between experimental and theoretical values was obtained, thus verifying the fulfillment of the reciprocity property in all the tested EATS devices. Despite minor differences, transmission properties are essentially 
the same for all the systems within the analyzed frequency range $(2000 \mathrm{~Hz})$, which clearly exceeds the usual range considered in an internal combustion engine exhaust [13].

Since the influence on the transmission coefficients of changes in the excitation characteristics is negligible in the absence of a mean flow [13], the results in Figure 3 lead to conclude that the systems tested are reciprocal under isolated excitations. However, a superimposed mean flow involves convective transport affecting to the wave propagation velocity. As discussed in [13], this convective transport gives rise to a relevant shift in the argument of the transmission coefficients. This is confirmed in Figure 4, in which the effects on the direct and inverse transmission coefficients due to the superimposition of a mean flow are shown for DOC+DPF-1. The argument of the direct transmission coefficient $t_{12}$ is shifted to higher frequencies when the device is subject to a mean flow, whereas the argument of the inverse transmission coefficient $t_{21}$ is shifted towards lower frequencies. The reason is found in the opposite interaction between the mean flow velocity and the pulse propagation velocity. On one hand, both velocities have the same direction in the direct test, so that the transmission propagation velocity is increased with respect to the baseline case without superimposed mean flow. In contrast, mean flow and perturbation have opposite directions in the inverse test and consequently the transmission propagation velocity results lower than in the baseline case.

As a result, the arguments of the direct $\left(t_{12}\right)$ and inverse $\left(t_{21}\right)$ transmission coefficients are always shifted in the presence of a superimposed mean flow. Consequently, the reciprocity property is not fulfilled, this being more noticeable as the frequency increases. This is shown in Figure 5, which compares the argument of the experimental and theoretical (computed from Eq. (2)) inverse transmission coefficients as well as their modulus for the tested EATS in the presence of a superimposed mean flow. As expected from the experimental data shown in Figure 4, the theoretical argument of $t_{21}$ does not match the experimental value for any device. Nevertheless, negligible differences are obtained in the modulus, this being the most relevant information on the transmission response obtained from the analysis of the reciprocity property.

\section{Dissipative behaviour analysis}

It is well known that the aftertreatment systems have a dissipative behaviour that makes them an interesting complement to the mufflers [9]. Therefore, the conservativeness property in reciprocal systems, which implies the fulfillment of Eqs. (3) and (4) as derived in Appendix C, does not hold in these devices.

$$
\begin{gathered}
\left|r_{1}\right|=\left|r_{2}\right| \\
\left(\frac{Y_{1}}{Y_{2}}\right)^{2}\left|t_{12}\right|^{4}+\left|r_{1}\right|^{4}-2 \operatorname{Re}\left(\frac{Y_{1}}{Y_{2}} t_{12}^{2} r_{1}^{*} r_{2}^{*}\right)=1
\end{gathered}
$$

Figure 6 shows the experimental results obtained for the modulus of the direct and inverse reflection coefficients of the tested EATS devices, for the baseline excitation and for pressure pulses with different amplitude and duration. It is clearly observed that the direct and inverse reflection coefficients are substantially different in all the devices 
regardless of the excitation characteristics, whose only effect is a slight frequency shift [13]. From Eq. (3), this would indicate that the systems are not conservative.

The acoustic dissipation can be easily taken into account by considering how the incident wave gives rise to transmission, reflection and dissipation. Rearranging Eq. (4), the energy balance for a reciprocal conservative system can be expressed, in the direct case, as

$$
\frac{Y_{1}}{Y_{2}}\left|t_{12}\right|^{2}+\left|r_{1}\right|^{2}=1
$$

and in the inverse direction as:

$$
\frac{Y_{2}}{Y_{1}}\left|t_{21}\right|^{2}+\left|r_{2}\right|^{2}=1
$$

If the system is non-conservative, the incident energy, besides being transmitted and reflected, is also partly dissipated. Therefore, a dissipation term $(\delta)$ [26] can be added to the balance shown in Eq. (5) and Eq. (6) that can be defined for the two propagation directions as:

$$
\begin{aligned}
& \delta_{\text {dir }}^{2}=1-\frac{Y_{1}}{Y_{2}}\left|t_{12}\right|^{2}-\left|r_{1}\right|^{2} \\
& \delta_{\text {inv }}^{2}=1-\frac{Y_{2}}{Y_{1}}\left|t_{21}\right|^{2}-\left|r_{2}\right|^{2}
\end{aligned}
$$

Based on experimental data, Figure 7 shows the direct and inverse dissipation terms obtained as a function of the excitation amplitude. The results were obtained according to Eq. (7) and Eq. (8) applied to the tested DOC+DPF systems as well as for TWC-1. The pulse amplitude was varied from 80 mbar to 230 mbar. Although the devices are reciprocal, the dissipation terms in the direct and inverse directions are different, as expected from the differences between $\left|r_{1}\right|$ and $\left|r_{2}\right|$ shown in Figure 6. Additionally, the dissipation behaviour is not significantly affected by the amplitude of the pressure pulses, with only some minor local deviations that could be attributed to measurement uncertainties.

As shown in Section 3, the convective transport caused by a superimposed mean flow only affects the relationship between the arguments of $t_{21}$ and $t_{12}$ but maintaining the equality between their modules. Since only the modulus is used to determine the dissipation terms, Eq. (7) and Eq. (8) can be applied with a superimposed mean mass flow. Experimental results are shown in Figure 8, where they are compared against the baseline case without superimposed mean flow. As in the case of a variation in the excitation amplitude, the superimposition of a mean flow does not have any impact on the dissipation behaviour of the different devices, neither in the direct nor in the inverse direction.

Therefore, as dissipation appears to be a property specific of each device and essentially independent on the operating conditions, the modulus of the direct and inverse reflection coefficients can be related to dissipation as:

$$
\left|r_{2}\right|^{2}=\left|r_{1}\right|^{2}+\delta_{\mathrm{dir}}^{2}-\delta_{\mathrm{inv}}^{2}
$$


One particular result of Eq. (9) is obtained for reciprocal non-conservative systems with equal dissipation terms in the direct and inverse directions. Under these conditions Eq. (9) simplifies to Eq. (3), i.e. the modulus of the direct and inverse reflection coefficient coincide. However, this is not the case in any of the devices tested.

Eq. (9) was applied to DOC+DPF systems and TWC-1 to calculate the $r_{2}$ modulus from the direct reflection coefficient and the dissipation terms. The experimental values obtained from the application of Eq. (7) and Eq. (8) to the baseline case (150 mbar in amplitude and $14 \mathrm{~ms}$ in duration without mean flow) were used as dissipation terms for every device. Figure 9 shows the predicted results compared against experimental data. Although some differences may be noticed, the accuracy of the results is good and consistent. Thus, by applying the proposed procedure it is possible to capture the main features of the reflection response of all the devices under a great variety of operating conditions.

\section{Simplified methodology for acoustic response prediction}

The use of reciprocity and dissipation properties makes it possible to simplify the acoustic characterization of aftertreatment systems. In this context, a modification of the methodology described in [13] for the prediction of acoustic properties is proposed that allows reducing the mount of fluid-dynamic modelling work. According to the flow-chart shown in Figure 10, the proposed methodology combines experimental and computational data to take into account the effect on the acoustic response of variations in the fluid-dynamic field.

The first step is to obtain the response of the system under direct and inverse excitations in the impulse test rig, exciting it in an arbitrary operating point considered as the baseline point. This procedure is summarized in the box labelled Experimental in Figure 10. The objective is to parameterize the scattering matrix coefficients, in particular $r_{2}$ and $t_{12}$, for the baseline point in terms of the addition of two Fourier series, as described in detail in [13]:

$$
F_{b, j}(f)=a_{0 j}+\sum_{k=1}^{n}\left(a_{k, j} \cos \left(k \tau_{b, j, \exp } f\right)+b_{k, j} \sin \left(k \tau_{b, j, \exp } f\right)\right)+a_{0 j}^{\prime}+\sum_{k=1}^{n}\left(a_{k, j}^{\prime} \cos \left(k \tau_{b, j, \exp }^{\prime} f\right)+b_{k, j}^{\prime} \sin \left(k \tau_{b, j, \exp }^{\prime} f\right)\right)
$$

In Eq $(10), F_{b, j}(f)$ represents the parameterized real or imaginary part of the scattering matrix coefficients (indexed by subscript $j$ ) obtained experimentally for the baseline operating point $(b)$. The terms $a_{k, j}$ and $b_{k, j}$ are the main Fourier series constants, which are specific to every device. Finally, $\tau_{b, j \text { exp }}$ is the characteristic period in the main Fourier series for the baseline operating point. In particular, this parameter is dependent on the operating conditions and the one representing the change in the acoustic response of the device as a function of the operating conditions [13]. The terms $a_{k, j}^{\prime}, b_{k, j}^{\prime}$ and $\tau_{b, j \text { exp }}^{\prime}$ correspond to the Fourier series that parameterizes the deviation between the experimental data and the main Fourier series. In addition, the experimental data allow obtaining the dissipation terms, $\delta_{\text {dir }}$ and $\delta_{\text {inv }}$, which are specific properties of each device and independent on the operating conditions, as discussed in Section 4.

Once the acoustic response of the device is characterized, a gas dynamic model is used to reproduce the experimental response in the baseline operating point and to predict the response of the device under different operating 
conditions. The modelling work is summarized in the box labelled Modelling in Figure 10. As discussed in [13], the only objective of this modelling work is to capture the wave propagation velocity in the time domain. However, in this new approach, the reciprocity and dissipation properties of the aftertreatment systems allow the gas dynamic modelling to be carried out only in one direction, for instance to calculate $r_{1}$ and $t_{12}$. This differs from the methodology proposed originally in [13], where both the direct and inverse responses were modelled.

The direct elements of the scattering matrix, i.e. $r_{1}$ and $t_{12}$, calculated by the gas dynamic model for the baseline and the operating point whose response is to be predicted, are subsequently parameterized by a Fourier series. Regardless of the accuracy of the model in the frequency domain, gas dynamic modelling is able to predict the shift in the scattering matrix coefficients due to a change in the characteristic propagation velocity between operating points. In this way, the ratio of the modelled characteristic periods obtained for the baseline (b) and modelled (p) points, i.e. $\tau_{b, j \text {,mod }}$ and $\tau_{p, j \text {,mod }}$, is representative of the actual one [13]:

$$
\pi_{p, j}=\frac{\tau_{p, j, \exp }}{\tau_{b, j, \exp }}=\frac{\tau_{p, j, \bmod }}{\tau_{b, j, \bmod }}
$$

Therefore, the characteristic period for the modelled point $\left(\tau_{p, j, \mathrm{pr}}\right)$ can be computed from Eq. (11), and the combined with the main Fourier series constants and with the deviation Fourier series to predict the direct scattering matrix elements for the modelled operating point (p). Once these terms are known, the reciprocity and dissipation properties of the device can be applied to determine the modulus of the inverse quantities $r_{2}$ and $t_{21}$. This is summarized in the box labelled Reciprocity and Dissipation Application in Figure 10.

This simplified methodology was applied to the prediction of the scattering matrix terms of TWC-1 when excited by a pressure pulse of 150 mbar in amplitude with a superimposed mean flow of $170 \mathrm{~kg} / \mathrm{h}$. Results are shown in Figure 11, where very good agreement between experimental data and modelled results can be observed up to $800 \mathrm{~Hz}$ in the transmission coefficients and up to $1500 \mathrm{~Hz}$ in the reflection coefficients. Above these frequencies, the prediction is still able to capture the response of the device but with some oscillations around the experimental value. The quality of these results is confirmed by the prediction of the transmission loss, both in the direct and inverse directions. As shown in Figure 12, the results were very accurate up to $800 \mathrm{~Hz}$, the proposed methodology being able to reproduce the qualitative response of the TWC-1 in the whole frequency range represented.

\section{Summary and conclusions}

A discussion on the fulfillment of acoustic reciprocity and conservativeness when applied to exhaust aftertreatment systems for use in internal combustion engines was conducted in this work. Acoustic reciprocity was defined for a two-port system in terms of the scattering matrix properties. The relation between the inverse and direct transmission coefficients through the ratio of the inlet to the outlet characteristic impedance was shown. If the system is also conservative, then the modules of the direct and inverse reflection coefficients are equal. 
The fulfillment of these properties in commercial exhaust aftertreatment systems was checked. Experimental data were obtained in an impulse test rig in which the devices were subject to isolated excitations of different amplitude, considering also the influence of a superimposed mean flow. Reciprocity was shown to be fulfilled in all the devices tested. In the absence of a superimposed mean flow this property is accomplished, both in argument and modulus, by the transmission coefficients regardless of the characteristics of the excitation. On the contrary, reciprocity does not hold for the argument of the transmission coefficients when a mean flow is superimposed on the impulsive excitation, because of the different convective effects occurring in the direct and the inverse directions. Nevertheless, the modulus of the transmission coefficient is still reciprocal under these operating conditions. Therefore, once the transmission coefficients are known in one direction of excitation, either from experimental or modelled data, it is then possible to predict easily the transmission coefficients in the other direction.

With respect to conservativeness, is has been demonstrated that, as well-known in the literature, the response of the devices involves dissipative characteristics. Nevertheless, given that the devices are reciprocal, an expression to compute both the direct and inverse dissipation terms from experimental data was derived. In turn, the modulus of the reflection coefficient in one direction can be determined by the modulus in the opposite direction and the two dissipation terms. Based on these ideas, it has been shown that for all the exhaust aftertreatment systems tested the dissipation terms are almost independent of the excitation and the mean flow. Therefore, once they were experimentally determined for some baseline conditions and knowing, either from experimental or modelled data, the reflection coefficient in one direction of excitation, the reflection coefficient in the other direction could be also determined. These results reveal great potential for their use in characterization processes relying on the reciprocity and dissipative behaviour of the elements under study. Once the system response is described in some reference conditions, the basis of its response under different operating conditions in the inverse direction can be directly obtained its characterization in the direct direction, and vice versa. This capability can be used as a consistency and reliability check for different features of experimental or numerical procedures, or be driven to the simplification of predictive tools, thus reducing the experimental and modelling workload. Concerning this case, the described approach has been included as a part of a methodology based on the impulse method aiming at predicting the influence of changes in the fluid-dynamic operating conditions on the acoustic response of exhaust aftertreatment systems. The application of reciprocity and dissipation relations provides an accurate prediction of the acoustic response relying only in the data provided for some arbitrary baseline test and the time domain modelling of for the direct excitation at the target operating conditions.

\section{Acknowledgements}

This research has been partially supported by FEDER and the Government of Spain through project TRA201679185-R. Additionally, the Ph.D. student Enrique José Sanchis has been funded by a grant from Universitat Politècnica de València with reference FPI-2016-S2-1355. 


\section{Appendix A: Transfer and scattering matrix definitions}

The scattering matrix of a two-port system is defined in the frequency domain as the matrix that provides the reflected sound pressure at the inlet and outlet sections of the device as a function of the incident pressure at those sections, according to

$$
\left[\begin{array}{c}
P_{1}^{-} \\
P_{2}^{+}
\end{array}\right]=\left[\begin{array}{ll}
r_{1} & t_{12} \\
t_{21} & r_{2}
\end{array}\right]\left[\begin{array}{c}
P_{1}^{+} \\
P_{2}^{-}
\end{array}\right]
$$

where $P$ is the sound pressure spectrum at inlet (1) and outlet (2) respectively, whilst superscripts + and - refer to forwards and backwards wave components. Therefore, the scattering matrix terms $r_{1}$ and $r_{2}$ represent the reflection coefficients whilst $t_{12}$ and $t_{21}$ are the transmission coefficients under direct (inlet to outlet, i.e. 12) and inverse (outlet to inlet, i.e. 21) excitations.

Eq. (A.1) can be rearranged to relate the inlet and outlet wave components by means of the transmission matrix. The elements of the transmission matrix can be written in terms of the reflection and transmission coefficients of the system, as shown in Eq. (A.2):

$$
\left[\begin{array}{c}
P_{1}^{+} \\
P_{1}^{-}
\end{array}\right]=\left[\begin{array}{cc}
H_{11}=t_{12}^{-1} & H_{12}=-\frac{r_{2}}{t_{12}} \\
H_{21}=\frac{r_{1}}{t_{12}} & H_{22}=t_{21}-\frac{r_{2} r_{1}}{t_{12}}
\end{array}\right]\left[\begin{array}{c}
P_{2}^{+} \\
P_{2}^{-}
\end{array}\right]
$$

The transfer matrix $\mathbf{T}$, which relates the acoustic pressure $(P)$ and mass velocity $(V)$ between inlet and outlet interfaces of a two-port system, is defined as [4]:

$$
\left[\begin{array}{c}
P_{1} \\
V_{1}
\end{array}\right]=\left[\begin{array}{ll}
T_{11} & T_{12} \\
T_{21} & T_{22}
\end{array}\right]\left[\begin{array}{c}
P_{2} \\
V_{2}
\end{array}\right]
$$

Additionally, the relation between wave components and acoustic pressure and mass velocity can be written in compact matrix form as

$$
\left.\begin{array}{c}
P=P^{+}+P^{-} \\
V=\frac{1}{Y}\left(P^{+}-P^{-}\right)
\end{array}\right\} \Rightarrow\left[\begin{array}{c}
P \\
V
\end{array}\right]=\underbrace{\left[\begin{array}{cc}
1 & 1 \\
Y^{-1} & -Y^{-1}
\end{array}\right]}_{\mathbf{Y}}\left[\begin{array}{c}
P^{+} \\
P^{-}
\end{array}\right]
$$

where $\mathbf{Y}$ is the characteristic impedance matrix. According to Munjal [4], any influence of mean flow or pulse amplitude on the characteristic impedance $Y$ would arise as a complex correction term associated with friction losses. However, such term would be significant only at Mach numbers much higher than those considered in this work, so that the no flow expression of $Y$ is considered:

$$
Y=\frac{a}{A}
$$


Eq. (A.5) is consistent with the choice of pressure and mass velocity fluctuations as variables, i.e. $a$ is the speed of sound and $A$ represents the cross-sectional area.

Substitution of Eq. (A.4) into Eq. (A.2) gives

$$
\underbrace{\frac{1}{2}\left[\begin{array}{cc}
1 & Y_{1} \\
1 & -Y_{1}
\end{array}\right]}_{\mathbf{Y}_{\text {in }}^{-1}}\left[\begin{array}{c}
P_{1} \\
V_{1}
\end{array}\right]=\underbrace{\left[\begin{array}{ll}
H_{11} & H_{12} \\
H_{21} & H_{22}
\end{array}\right]}_{\mathbf{H}} \underbrace{\frac{1}{2}\left[\begin{array}{cc}
1 & Y_{2} \\
1 & -Y_{2}
\end{array}\right]}_{\mathbf{Y}_{\text {out }}^{-1}}\left[\begin{array}{c}
P_{2} \\
V_{2}
\end{array}\right]
$$

and rearranging Eq. (A.6) and comparing with Eq. (A.3), the transfer matrix can be finally expressed as:

$$
\mathbf{T}=\mathbf{Y}_{\mathrm{in}} \mathbf{H} \mathbf{Y}_{\text {out }}^{-1}
$$

\section{Appendix B: Reciprocity}

Reciprocity is traditionally defined by the condition that the transfer matrix determinant is equal to 1 [22], i.e:

$$
\Delta=T_{11} T_{22}-T_{12} T_{21}=1
$$

Taking into account Eq. (A.7), this condition may be readily expressed in terms of the determinant of the transmission matrix as:

$$
|\mathbf{H}|=\left|\mathbf{Y}_{\text {in }}^{-1}\right| \Delta\left|\mathbf{Y}_{\text {out }}\right|=\frac{Y_{1}}{Y_{2}}
$$

Then, expressing the transmission matrix elements in terms of the transmission and reflection coefficients, as shown in Eq. (A.2), the reciprocity condition can be rewritten as

$$
\frac{Y_{1}}{Y_{2}}=H_{11} H_{22}-H_{12} H_{21}=\frac{1}{t_{12}}\left(t_{21}-\frac{r_{2} r_{1}}{t_{12}}\right)+\frac{r_{2} r_{1}}{t_{12}^{2}}=\frac{t_{21}}{t_{12}}
$$

and after some algebra one finally gets

$$
t_{21}=\frac{Y_{1}}{Y_{2}} t_{12}
$$

In this way, reciprocity is expressed as the condition that the transmission coefficients in the direct and inverse directions are proportional, the constant of proportionality being the ratio of the characteristic impedances.

\section{Appendix C: Reciprocity and conservativeness}

Conservativeness is expressed in terms of the transfer matrix elements as [22]:

$$
T_{11}^{*}=\frac{T_{11}}{\Delta} \quad T_{12}^{*}=-\frac{T_{12}}{\Delta} \quad T_{21}^{*}=-\frac{T_{21}}{\Delta} \quad T_{22}^{*}=\frac{T_{22}}{\Delta}
$$


Rearranging and expanding Eq. (A.7), the transmission matrix coefficients can be written as

$$
\begin{aligned}
& H_{11}=\frac{1}{2}\left(T_{11}+\frac{T_{12}}{Y_{2}}+Y_{1} T_{21}+\frac{Y_{1}}{Y_{2}} T_{22}\right) \\
& H_{12}=\frac{1}{2}\left(T_{11}-\frac{T_{12}}{Y_{2}}+Y_{1} T_{21}-\frac{Y_{1}}{Y_{2}} T_{22}\right) \\
& H_{21}=\frac{1}{2}\left(T_{11}+\frac{T_{12}}{Y_{2}}-Y_{1} T_{21}-\frac{Y_{1}}{Y_{2}} T_{22}\right) \\
& H_{22}=\frac{1}{2}\left(T_{11}-\frac{T_{12}}{Y_{2}}-Y_{1} T_{21}+\frac{Y_{1}}{Y_{2}} T_{22}\right)
\end{aligned}
$$

If the system is reciprocal, i.e. $\Delta=1$, the conjugates of the transmission matrix elements can be directly expressed in terms of the transfer matrix elements and the characteristic impedances as

$$
\begin{aligned}
& H_{11}^{*}=\frac{1}{2}\left(T_{11}-\frac{T_{12}}{Y_{2}}-Y_{1} T_{21}+\frac{Y_{1}}{Y_{2}} T_{22}\right) \\
& H_{12}^{*}=\frac{1}{2}\left(T_{11}+\frac{T_{12}}{Y_{2}}-Y_{1} T_{21}-\frac{Y_{1}}{Y_{2}} T_{22}\right) \\
& H_{21}^{*}=\frac{1}{2}\left(T_{11}-\frac{T_{12}}{Y_{2}}+Y_{1} T_{21}-\frac{Y_{1}}{Y_{2}} T_{22}\right) \\
& H_{22}^{*}=\frac{1}{2}\left(T_{11}+\frac{T_{12}}{Y_{2}}+Y_{1} T_{21}+\frac{Y_{1}}{Y_{2}} T_{22}\right)
\end{aligned}
$$

Considering the definition of the transmission matrix elements given in Eq. (C.2), this finally yields:

$$
H_{12}=H_{21}^{*}
$$

$$
H_{11}=H_{22}^{*}
$$

On one hand, Eq. (C.4) and its conjugate $\left(H_{12}^{*}=H_{21}\right)$ can be expressed in terms of the transmission and reflection coefficients as indicated by Eq. (A.2). The resulting expression can be rearranged to obtain the relationship between the reflection coefficients:

$$
\left|r_{1}\right|=\left|r_{2}\right|
$$

On the other hand, Eq. (C.5) and its conjugate $\left(H_{11}^{*}=H_{22}\right)$ can be expressed making use of the definition of the scattering matrix elements shown in Appendix A. Multiplying the resulting expressions and applying the reciprocity property, which is defined in Eq. (B.4), Eq. (C.7) is obtained:

$$
\left(\frac{Y_{1}}{Y_{2}}\right)^{2}\left|t_{12}\right|^{4}+\left|r_{1}\right|^{4}-2 \operatorname{Re}\left(\frac{Y_{1}}{Y_{2}} t_{12}^{2} r_{1}^{*} r_{2}^{*}\right)=1
$$

Therefore, a conservative reciprocal system fulfills Eq. (C.6) and Eq. (C.7).

\section{References}

[1] Regulation (EC) no 715/2007 of the European Parliament and of the Council of 20 June 2007 on type approval of motor vehicles with respect to emissions from light passenger and commercial vehicles (Euro 5 and Euro 6) and on access to vehicle repair and maintenance information, Official Journal of the European Union (June 2007). 
[2] Control of air pollution from motor vehicles: Tier 3 motor vehicle emission and fuel standards, Proposed Rules, Federal Register, vol.78, No 98 (May 2013)

[3] T.V. Johnson, Review of vehicular emissions trends, in: SAE Technical Paper 2015-01-0993, 2015.

[4] M. Munjal, Acoustics of ducts and mufflers, 2nd ed. John Wiley \& Sons, New York, 2014.

[5] E. Dokumaci, Sound transmission in narrow pipes with superimposed uniform mean flow and acoustic modelling of automobile catalytic converters, J. Sound Vib. 182 (5) (1995) 799-808.

[6] X. Hua, D.W. Herrin, T.W. Wu, T. Elnady, Simulation of diesel particulate filters in large exhaust systems, Appl. Acoust. 74 (2013) $1326-1332$.

[7] J.M. Desantes, A.J. Torregrosa, A. Broatch, H. Climent, Silencing capabilities of non-silencer elements: an underused potential?, in: 4th Styrian Noise, Vibration \& Harshness Congress, Graz, Austria, November 15-17, 2006.

[8] S. Allam, M. Åbom, Sound propagation in an array of narrow porous channels with application to Diesel particulate filters, J. Sound Vib. 291 (2006) 882-901.

[9] A. Selamet, V. Easwaran, J.M. Novak, R.A. Kach, Wave attenuation in catalytic converters: reactive versus dissipative effects, J. Acoust. Soc. Am. 103 (2) (1998) 935-943.

[10] S. Allam, M. Åbom, Acoustic modelling and testing of Diesel particulate filters, J. Sound Vib. 288 (2005) $255-273$.

[11] J. Hicks, W. Hill, A. Kotrba, DPF acoustic performance: an evaluation of various substrate materials and soot conditions, in: SAE Technical Paper 2011-01-2198, 2011.

[12] A.J. Torregrosa, J.R. Serrano, P. Piqueras, O. García-Afonso, Experimental and computational approach to the transient behaviour of wallflow diesel particulate filters, Energy 119 (2016) 887-900.

[13] A.J. Torregrosa, F.J. Arnau, P. Piqueras, E.J. Sanchis, H. Tartoussi, Phenomenological methodology for assessing the influence of flow conditions on the acoustic response of exhaust aftertreatment systems, J. Sound Vib. 396 (2017) 289-306.

[14] G. Montenegro, G. Onorati, A. Della Torre, The prediction of silencer acoustical performances by 1D, 1D-3D and quasi-3D non-linear approaches, Comput. Fluids 71 (2013) 208-223.

[15] K. Peat, A first approximation to the effects of mean flow on sound propagation tin capillary tubes, J. Sound Vib. 175 (1994) $475-489$.

[16] R.J. Astley, A. Cummings, Wave propagation in catalytic converters: formulation of the problem and finite element solution scheme, J. Sound Vib. 88 (5) (1995) 635-657.

[17] C. Jiang, T.W. Wu, M.B. Xu, C.Y.R. Cheng, BEM modeling of mufflers with diesel particulate filters and catalytic converters, Noise Control Eng. J. 58 (3) (2010) 243-250.

[18] F. Payri, J.M. Desantes, A. Broatch, Modified impulse method for the measurement of the frequency response of acoustic filters to weakly nonlinear transient excitations, J. Acoust. Soc. Am. 107 (2) (2000) 731-738.

[19] H. von Helmholtz, Handbook of physiological optics, Leopold Voss, Leipzig, 1867.

[20] J.W. Strutt, The theory of sound, Macmillan and Co., London, 1877.

[21] V. Pagneux, A. Maurel, Scattering matrix properties with evanescent modes for waveguides in fluids and solids, J. Acoust. Soc. Am. 116 (2004) 1913-1920.

[22] V.H. Gupta, On independence of reciprocity, symmetry and conservativeness of one-dimensional linear systems, J. Sound Vib. 179 (3) (1995) $547-552$.

[23] V. Easwaran, V.H. Gupta, M. Munjal, Relationship between the impedance matrix and the transfer matrix with specific reference to symmetric, reciprocal and conservative systems, J. Sound Vib. 161 (3) (1993) 515-525.

[24] F.K. Bannister, G.F. Mucklow, Wave action following sudden release of compressed gas from a cylinder, P. I. Mech. Eng. 159 (1948) $269-287$.

[25] F. Payri, J.M. Desantes, A.J. Torregrosa, Acoustic boundary condition for unsteady one-dimensional flow calculations, J. Sound Vib. 188 (1) (1995) 85-110.

[26] R. Kabral, F. Auriemma, M. Knutsson, M. Åbom, A new type of compact silencer for high frequency noise, Proceedings of the 9th International Conference of DAAAM Baltic Industrial Engineering, Tallinn, Estonia, April 24-26, 2014. 


\section{List of Tables}

- Table 1.- Main geometric parameters of the characterized EATS.

\section{List of Figures}

- Figure 1.- Schematic setup and operating principle of the impulse test rig.

- Figure 2.- Example of pressure wave decomposition in impulse test rig. Pressure pulse $150 \mathrm{mbar}$ in amplitude and $14 \mathrm{~ms}$ in duration in DOC+DPF-2: (a) direct test, (b) inverse test.

- Figure 3.- Comparison between experimental and theoretical inverse transmission coefficients applying the reciprocity property. Pressure pulse 150 mbar in amplitude and $14 \mathrm{~ms}$ in duration in different EATS devices: (a) DOC+DPF-1, (b) TWC-1, (c) DOC+DPF-2.

- Figure 4.- Experimental transmission coefficients of DOC+DPF-1 corresponding to direct and inverse tests with a change in superimposed mass flow from $0 \mathrm{~kg} / \mathrm{h}$ to $170 \mathrm{~kg} / \mathrm{h}$ (Mach 0.055). (a) Argument of the direct transmission coefficient, (b) modulus of the direct transmission coefficient, (c) argument of the inverse transmission coefficient, (d) modulus of the inverse transmission coefficient.

- Figure 5.- Comparison between experimental and theoretical inverse transmission coefficients $\left(t_{21}\right)$ applying the reciprocity hypothesis to the superimposed mean mass flow case (170 kg/h (Mach 0.055)) in different EATS devices: (a) DOC+DPF-1, (b) TWC-1, (c) DOC+DPF-2.

- Figure 6.- Comparison between experimental reflection coefficients obtained in direct and inverse tests for different EATS devices: pressure pulse 150 mbar in amplitude and $14 \mathrm{~ms}$ in duration in (a) DOC+DPF-1, (b) DOC+DPF-2 and (c) TWC-1; pressure pulse 230 mbar in amplitude and $18 \mathrm{~ms}$ in duration in (d) DOC+DPF-1 and (e) DOC+DPF-2; and pressure pulse 80 mbar in amplitude and $11 \mathrm{~ms}$ in duration in (f) TWC-1.

- Figure 7.- Experimental dissipation terms of direct and inverse tests for different EATS devices as a function of pressure pulse amplitude: direct test in (a) DOC+DPF-1, (b) DOC+DPF-2 and (c) TWC-1; inverse test in (d) DOC+DPF-1, (e) DOC+DPF-2 and (f) TWC-1.

- Figure 8.- Experimental dissipation terms of direct and inverse tests corresponding to the superimposed mean mass flow $(170 \mathrm{~kg} / \mathrm{h}$ (Mach 0.055)) case in different EATS devices: direct test in (a) DOC+DPF-1, (b) DOC+DPF-2 and (c) TWC-1; inverse test in (d) DOC+DPF-1, (e) DOC+DPF-2 and (f) TWC-1.

- Figure 9.- Comparison between experimental and theoretical inverse reflection coefficients for different EATS devices from baseline dissipation terms. Application to cases with excitations of different amplitude and superimposed mean mass flow: w/o mean mass flow in (a) DOC+DPF-1, (b) DOC+DPF-2 and (c) TWC-1; with $170 \mathrm{~kg} / \mathrm{h}$ (Mach 0.055) in mean mass flow in (d) DOC+DPF-1, (e) DOC+DPF-2 and (f) TWC-1.

- Figure 10.- Flowchart of the simplified methodology for acoustic response prediction based on the application of reciprocity and dissipation properties. 
- Figure 11.- Comparison between experimental and modelled (a) $r_{1}$ modulus, (b) $t_{12}$ modulus, (c) $t_{21}$ modulus and (d) $r_{2}$ modulus of TWC-1. Pressure pulse of 150 mbar in amplitude and $14 \mathrm{~ms}$ in duration with superimposed mean mass flow $(170 \mathrm{~kg} / \mathrm{h})$.

- Figure 12.- Comparison between experimental and modelled (a) direct and (b) inverse transmission loss of TWC-1. Pressure pulse of $150 \mathrm{mbar}$ in amplitude and $14 \mathrm{~ms}$ in duration with superimposed mean mass flow $(170 \mathrm{~kg} / \mathrm{h})$.

Table 1: Main geometric parameters of the characterized EATS.

\begin{tabular}{ccccccc}
\hline \hline & & DOC-1 & DPF-1 & DOC-2 & DPF-2 & TWC-1 \\
\hline \hline$D$ & {$[\mathrm{~mm}]$} & 172 & 172 & 144 & 144 & 112 \\
$L$ & {$[\mathrm{~mm}]$} & 82 & 105 & 114 & 130 & 127 \\
$\alpha$ & {$[\mathrm{mm}]$} & 0.83 & 1.4 & 0.87 & 1.4 & 0.97 \\
$w_{w}$ & {$[\mathrm{~mm}]$} & 0.44 & 0.4 & 0.4 & 0.4 & 0.11 \\
$\sigma$ & {$[\mathrm{cpsi}]$} & 400 & 200 & 400 & 200 & 600 \\
\hline \hline
\end{tabular}

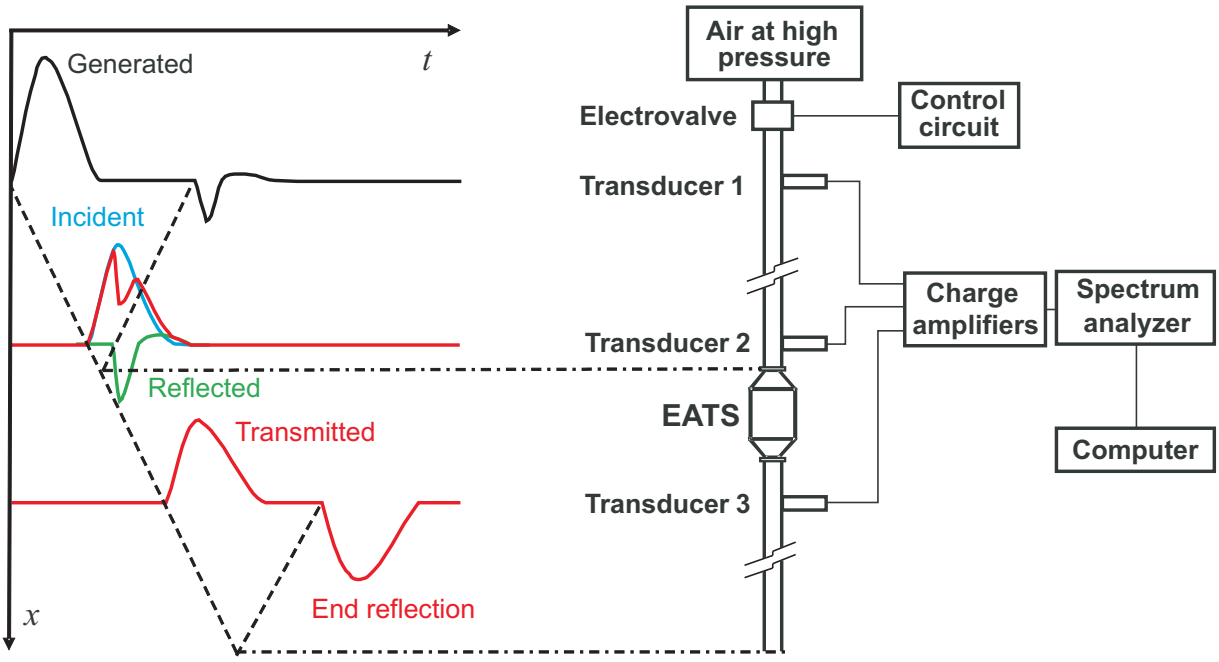

Figure 1: Schematic setup and operating principle of the impulse test rig 




Figure 2: Example of pressure wave decomposition in impulse test rig. Pressure pulse $150 \mathrm{mbar}$ in amplitude and $14 \mathrm{~ms}$ in duration in DOC+DPF-2: (a) direct test, (b) inverse test.
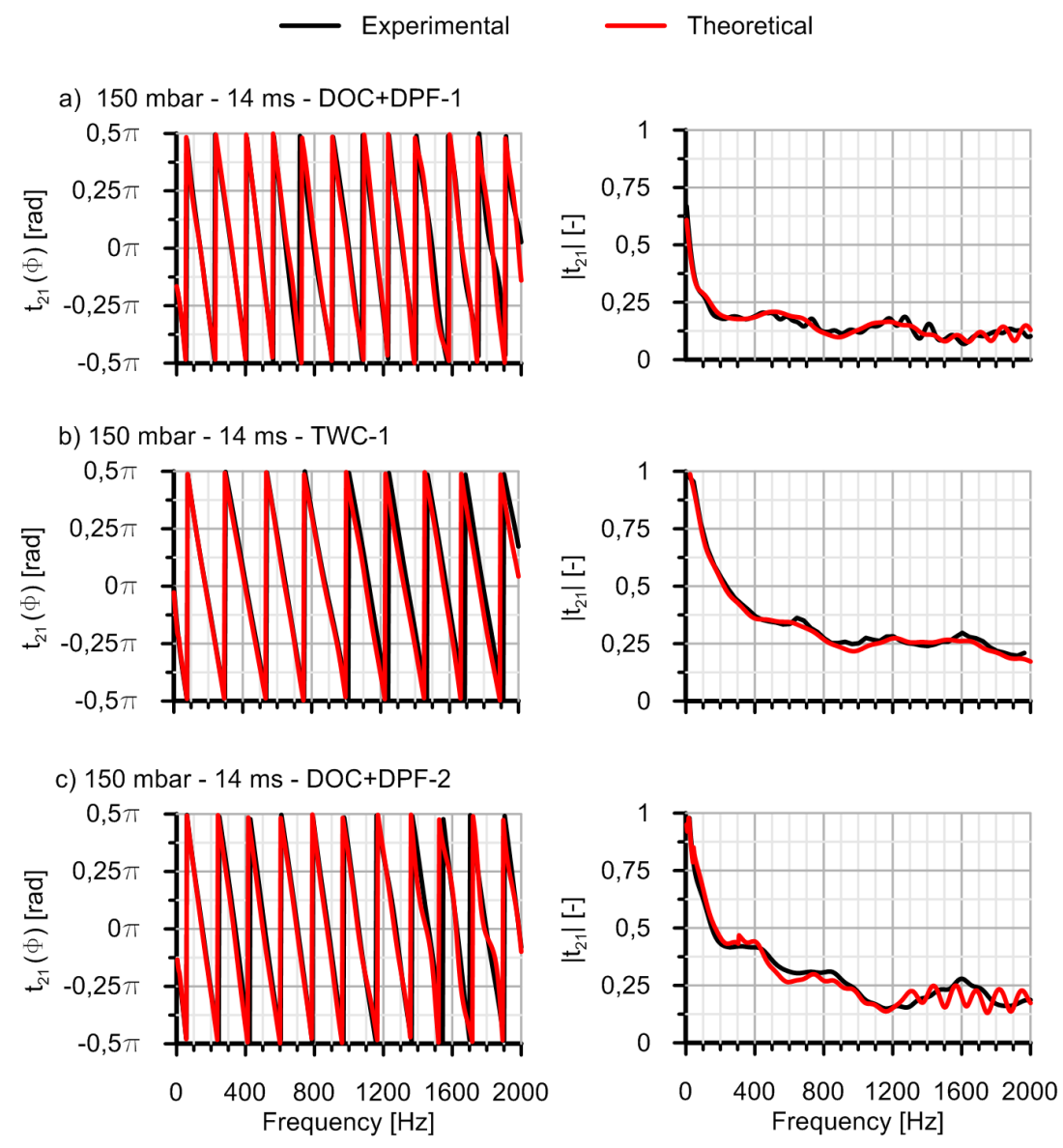

Figure 3: Comparison between experimental and theoretical inverse transmission coefficients applying the reciprocity property. Pressure pulse $150 \mathrm{mbar}$ in amplitude and $14 \mathrm{~ms}$ in duration in different EATS devices: (a) DOC+DPF-1, (b) TWC-1, (c) DOC+DPF-2. 
150 mbar - w/o mean flow

a)

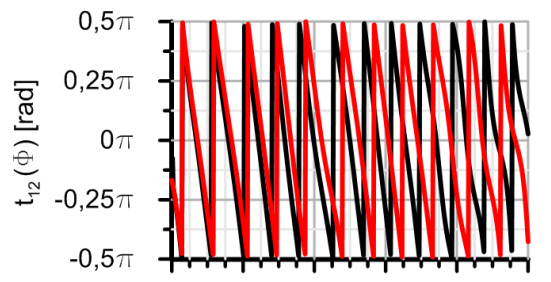

c)

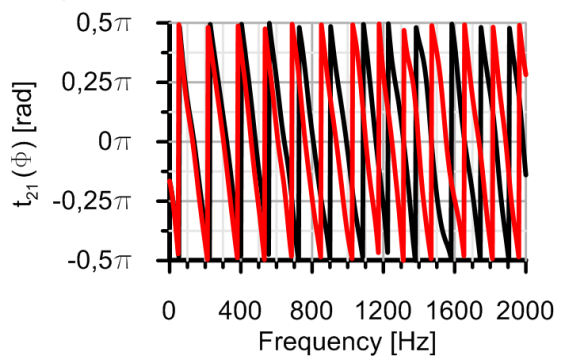

$150 \mathrm{mbar}-170 \mathrm{~kg} / \mathrm{h}$ mean flow

b)

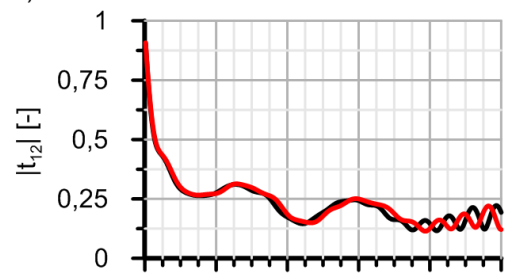

d)

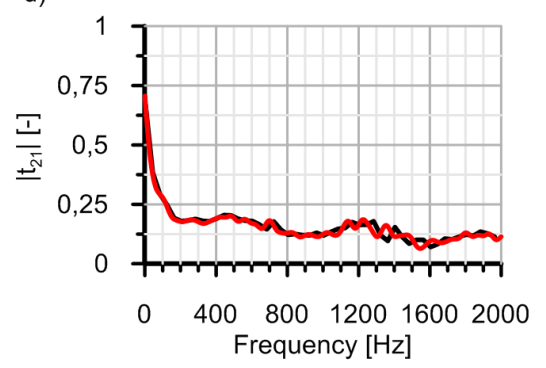

Figure 4: Experimental transmission coefficients of DOC+DPF-1 corresponding to direct and inverse tests with a change in superimposed mass flow from $0 \mathrm{~kg} / \mathrm{h}$ to $170 \mathrm{~kg} / \mathrm{h}$ (Mach 0.055). (a) Argument of the direct transmission coefficient, (b) modulus of the direct transmission coefficient, (c) argument of the inverse transmission coefficient, (d) modulus of the inverse transmission coefficient. 

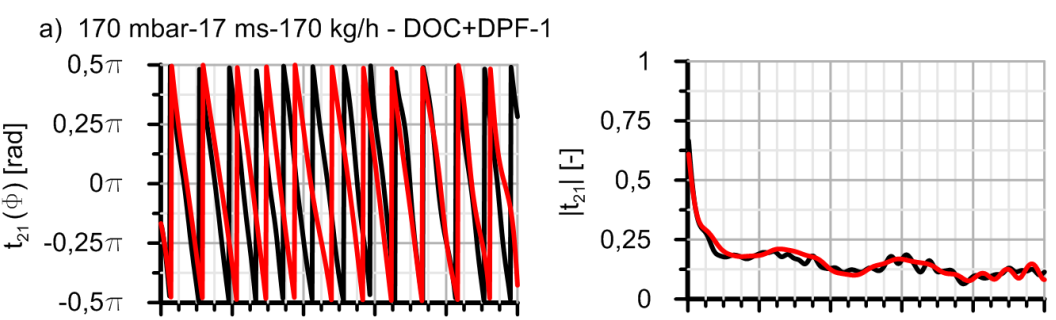

b) $170 \mathrm{mbar}-17 \mathrm{~ms}-170 \mathrm{~kg} / \mathrm{h}$ - TWC-1
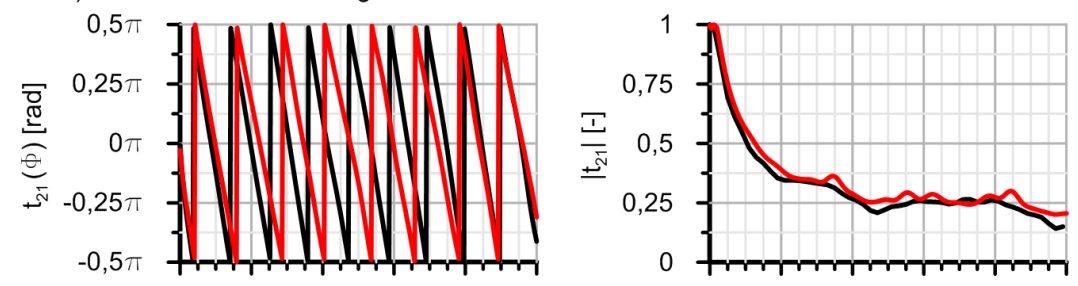

c) $170 \mathrm{mbar}-17 \mathrm{~ms}-170 \mathrm{~kg} / \mathrm{h}$ - DOC+DPF-2
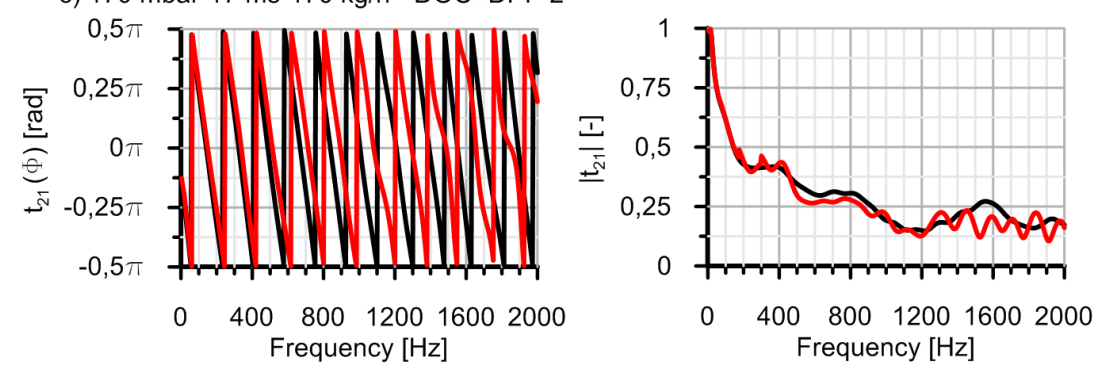

Figure 5: Comparison between experimental and theoretical inverse transmission coefficients $\left(t_{21}\right)$ applying the reciprocity hypothesis to the superimposed mean mass flow case (170 kg/h (Mach 0.055)) in different EATS devices: (a) DOC+DPF-1, (b) TWC-1, (c) DOC+DPF-2. 


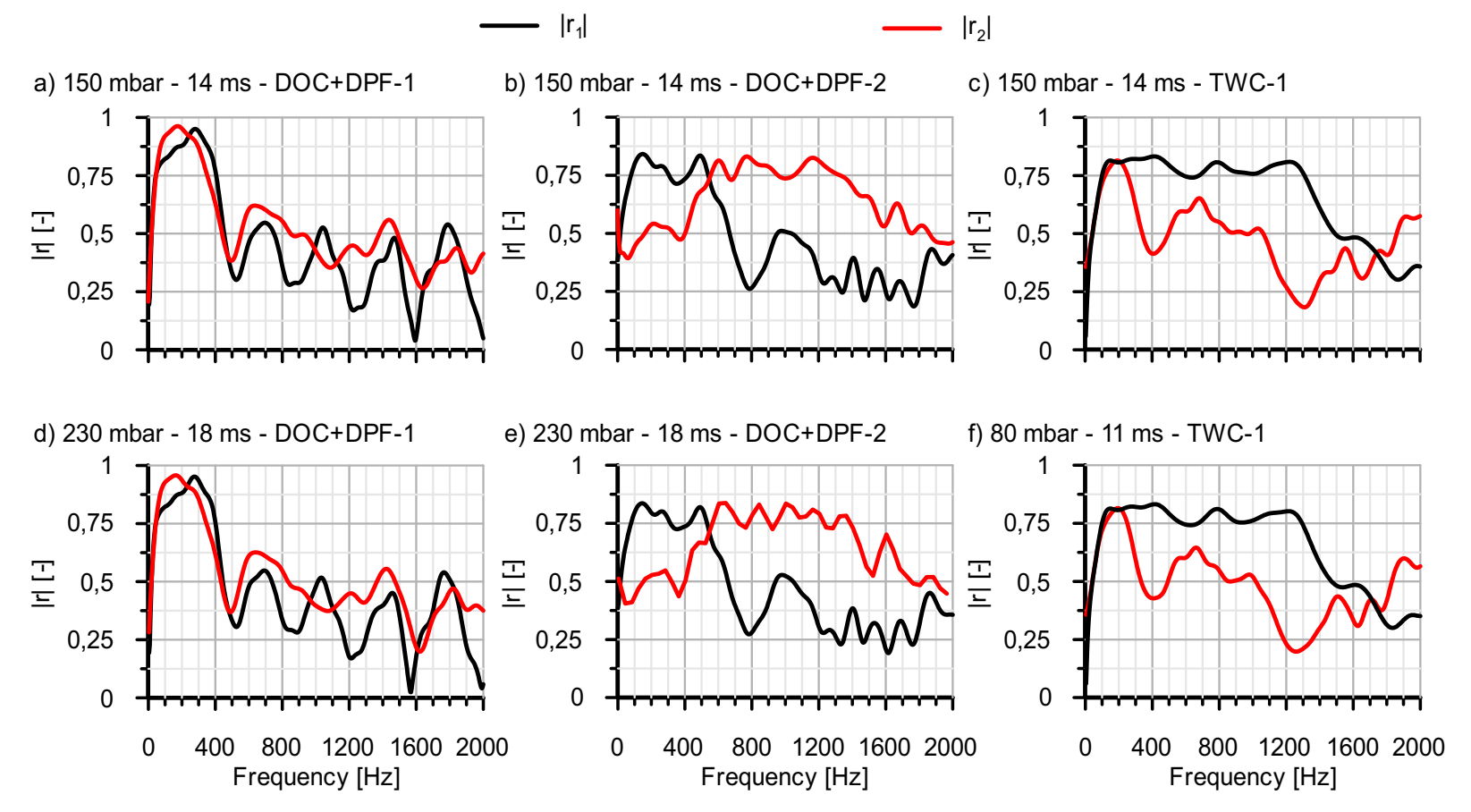

Figure 6: Comparison between experimental reflection coefficients obtained in direct and inverse tests for different EATS devices: pressure pulse 150 mbar in amplitude and $14 \mathrm{~ms}$ in duration in (a) DOC+DPF-1, (b) DOC+DPF-2 and (c) TWC-1; pressure pulse 230 mbar in amplitude and $18 \mathrm{~ms}$ in duration in (d) DOC+DPF-1 and (e) DOC+DPF-2; and pressure pulse 80 mbar in amplitude and $11 \mathrm{~ms}$ in duration in (f) TWC-1. 

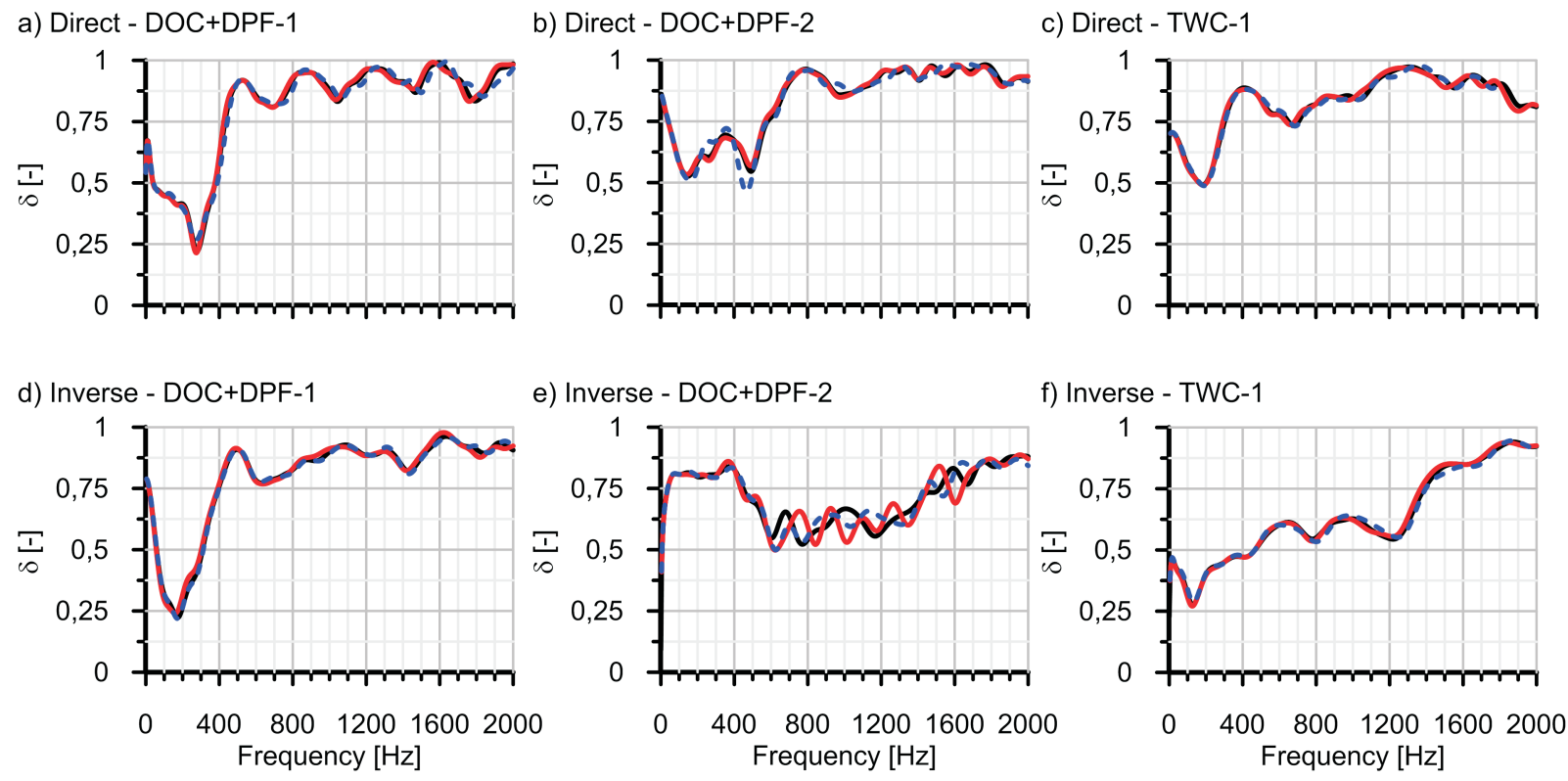

Figure 7: Experimental dissipation terms of direct and inverse tests for different EATS devices as a function of pressure pulse amplitude: direct test in (a) DOC+DPF-1, (b) DOC+DPF-2 and (c) TWC-1; inverse test in (d) DOC+DPF-1, (e) DOC+DPF-2 and (f) TWC-1. 
a) Direct - DOC+DPF-1

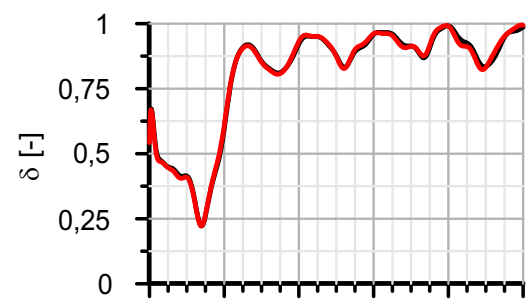

c) Direct - DOC+DPF-2

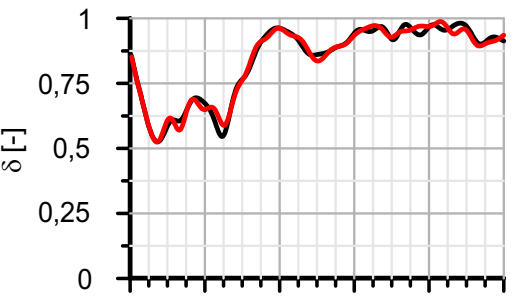

e) Direct - TWC-1

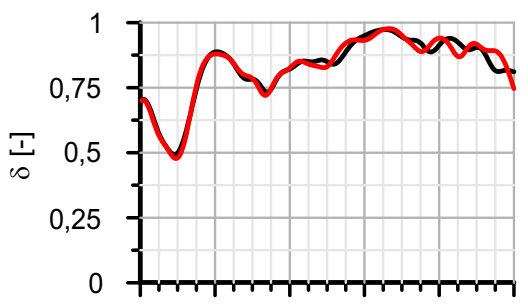

f) Inverse - TWC-1

d) Inverse - DOC+DPF-2

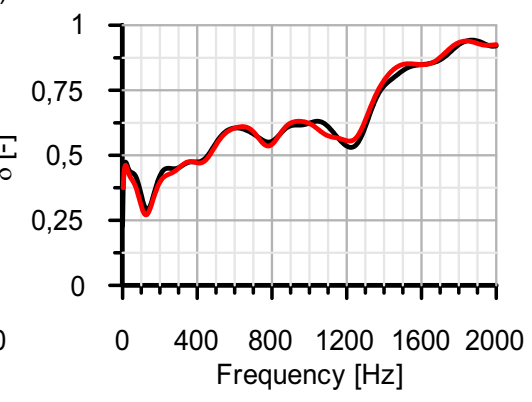



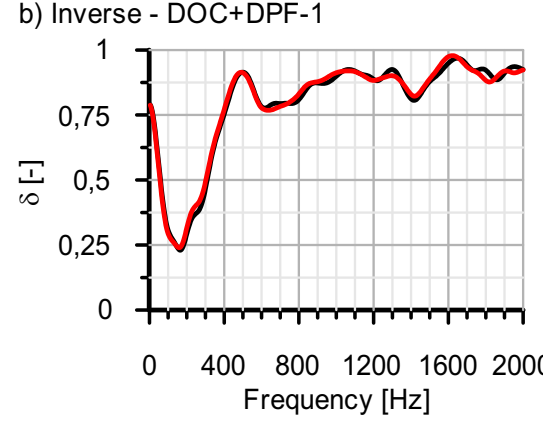

Figure 8: Experimental dissipation terms of direct and inverse tests corresponding to the superimposed mean mass flow (170 kg/h (Mach 0.055)) case in different EATS devices: direct test in (a) DOC+DPF-1, (b) DOC+DPF-2 and (c) TWC-1; inverse test in (d) DOC+DPF-1, (e) DOC+DPF-2 and (f) TWC-1. 
a) $230 \mathrm{mbar}-18 \mathrm{~ms}-0 \mathrm{~kg} / \mathrm{h}$ - DOC+DPF-1

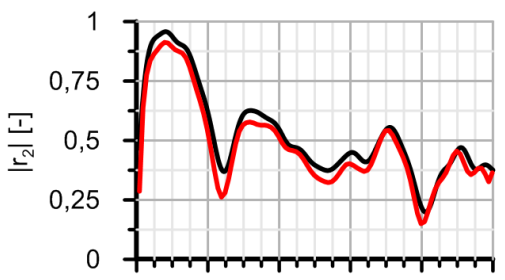

b) $230 \mathrm{mbar}-18 \mathrm{~ms}-0 \mathrm{~kg} / \mathrm{h}$ - DOC+DPF-2

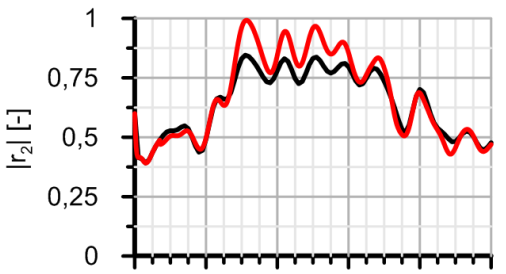

c) $230 \mathrm{mbar}-18 \mathrm{~ms}-0 \mathrm{~kg} / \mathrm{h}$ - TWC-1

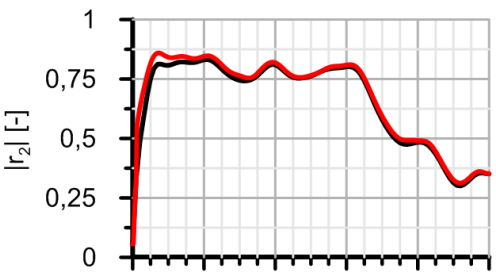

d) $170 \mathrm{mbar}-17 \mathrm{~ms}-170 \mathrm{~kg} / \mathrm{h}$ - DOC+DPF-1 e) $170 \mathrm{mbar}-17 \mathrm{~ms}-170 \mathrm{~kg} / \mathrm{h}$ - DOC+DPF-2 f) $170 \mathrm{mbar}-17 \mathrm{~ms}-170 \mathrm{~kg} / \mathrm{h}$ - TWC-1
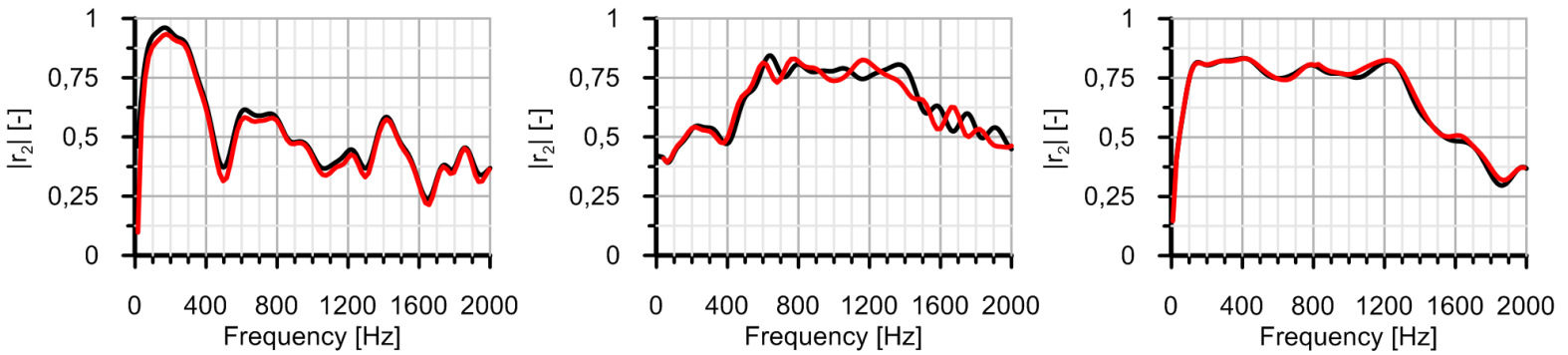

Figure 9: Comparison between experimental and theoretical inverse reflection coefficients for different EATS devices from baseline dissipation terms. Application to cases with excitations of different amplitude and superimposed mean mass flow: w/o mean mass flow in (a) DOC+DPF-1, (b) DOC+DPF-2 and (c) TWC-1; with $170 \mathrm{~kg} / \mathrm{h}$ (Mach 0.055) in mean mass flow in (d) DOC+DPF-1, (e) DOC+DPF-2 and (f) TWC-1. 




Figure 10: Flowchart of the simplified methodology for acoustic response prediction based on the application of reciprocity and dissipation properties. 
a)

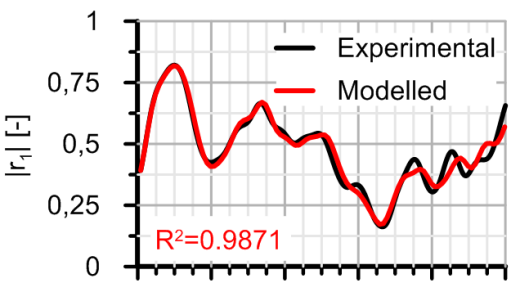

c)

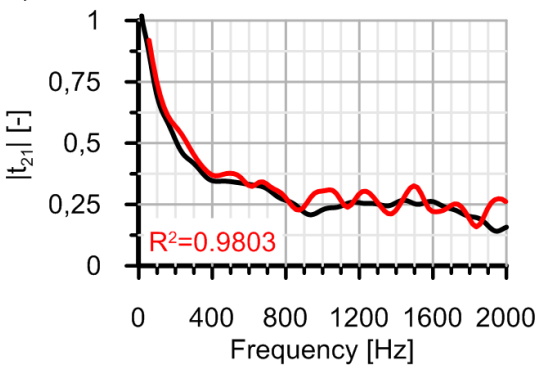

b)

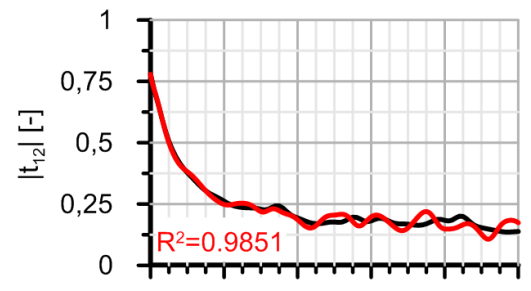

d)

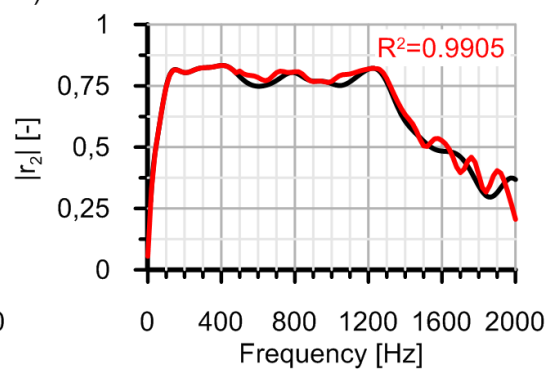

Figure 11: Comparison between experimental and modelled (a) $r_{1}$ modulus, (b) $t_{12}$ modulus, (c) $t_{21}$ modulus and (d) $r_{2}$ modulus of TWC-1. Pressure pulse of $150 \mathrm{mbar}$ in amplitude and $14 \mathrm{~ms}$ in duration with superimposed mean mass flow $(170 \mathrm{~kg} / \mathrm{h})$.

a)

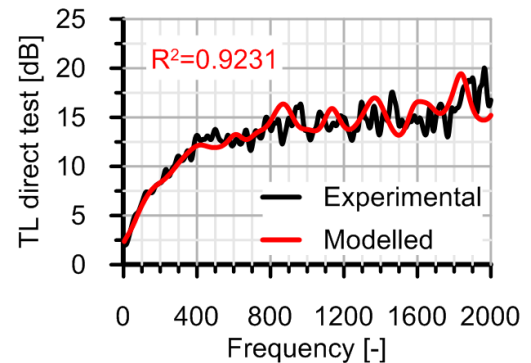

b)

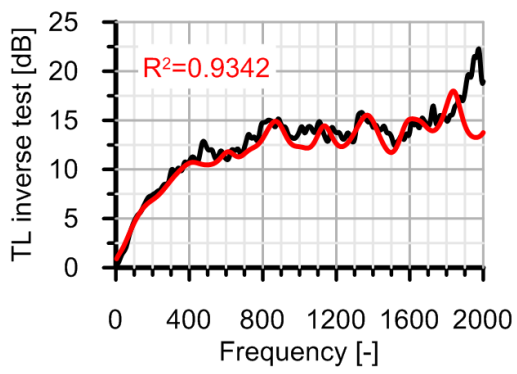

Figure 12: Comparison between experimental and modelled (a) direct and (b) inverse transmission loss of TWC-1. Pressure pulse of 150 mbar in amplitude and $14 \mathrm{~ms}$ in duration with superimposed mean mass flow $(170 \mathrm{~kg} / \mathrm{h})$. 